\title{
Control Oriented Model for Expander Cycle Scramjet
}

\author{
Zhelong Zhao ${ }^{1}$, Xianyu $\mathrm{Wu}^{1}$ \\ ${ }^{1}$ College of Aerospace Science and Engineering, National University of Defence Technology, Changsha 410073, China
}

\begin{abstract}
As a efficient and simple design, expander cycle is widely applied in LRE engineering, but it is seldomly used on scramjet research. In order to establish a complete mathematical model for expander cycle scramjet, a control-oriented model for expander cycle scramjet is proposed in this paper. This model consists of four major parts: combustor, cooling channel, turbo pump and nozzle and gives the result of pressure, temperature, mach number and velocity distribution of combustor and cooling channel and is capable of simulate both pure supersonic combustion mode and supersonic shock wave mode of the combustor. Each part is given by specific mathematical description, which contains the calculation of airflow, combustion, heat transfer and thermal cracking of kerosene. By putting all these parts together, a complete model is formed. This model is proposed to calculate the performance and condition of the engine precisely, comprehensively, swiftly and can be directly used in further study.
\end{abstract}

\section{Introduction}

Hypersonic airbreathing vehicle has shown its bright future in both military and civil uses. Because of its outstanding performance at the speed range from mach 5 to mach 10 , scramjet is thought to be the ideal propulsion system to achieve hypersonic airbreathing flight.

Comparing to other designs, expander cycle is more efficient, more reliable and has a simple structure. The working process of expander cycle scramjet is shown in fig.1. Centrifugal pump pumps cold fuel from the fuel tank to the turbo through cooling channel, within which the cold fuel transfer heat with combustor wall and becomes hot fuel. Hot fuel powers the turbine to drive the centrifugal pump. After flow out of the turbine, the fuel is injected into the combustor to mix with air and release heat after combustion.

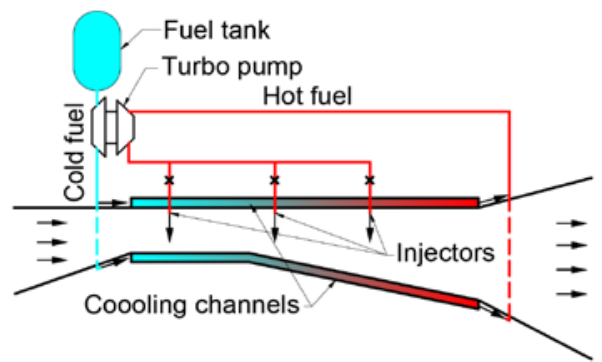

Figure1. Expander cycle scramjet

The design and application of expander cycle fit scramjet perfectly, but there are few research about expander cycle scramjet, and further research requires a model that gives expander cycle scramjet a complete mathematical description. In this paper, aiming on forming a self-holding system that contains the key factors of a kerosene based scramjet, a quasi-one dimensional model for expander cycle scramjet is proposed. This model can be used on analysis the working characteristics and predict the performance of expander cycle scramjet.

\section{Modelling}

\subsection{Modelling of combustor}

The condition of the airflow can be defined by its composition, pressure, velocity, temperature, density and mach number. Thus, by setting up and solving the governing equations of the control volume, we can picture the whole flow field of the combustor. The governing equations is set under the following assumptions: a, gas in the combustor is prefect gas which meets the ideal gas equation, $b$, air flow is steady flow, and the equation does not contain any time term, c, all parameters change only along $\mathrm{X}$ axis. Referring to [1], the governing equations is given by:

$$
\begin{aligned}
& (\rho+d \rho)(A+d A)(u+d u)=\rho A u+d \dot{m} \\
& \frac{1}{u} \frac{d u}{d x}=-\frac{1}{\rho u^{3}} \frac{d p}{d x}-\frac{2 C_{f}}{D}-\frac{(1-\varepsilon)}{\dot{m}} \frac{d \dot{m}}{d x} \\
& \mathrm{Cp} \frac{d T}{d x}=-\sum_{\mathrm{i}} h_{i} \frac{d Y_{i}}{d x}-u \frac{d u}{d x}+\frac{1}{\dot{m}} \sum_{\mathrm{i}}\left(h_{i} \frac{d \dot{m}_{i}}{d x}\right)_{\text {add }}-\frac{2 C_{f}\left(h_{a w}-h_{w}\right)}{D P_{r} \frac{2}{3}}-\frac{h_{t}}{\dot{m}} \frac{d \dot{m}}{d x} \\
& \frac{1}{p} \frac{d p}{d x}=\frac{1}{\rho} \frac{d \rho}{d x}+\frac{1}{T} \frac{d T}{d x}-\frac{1}{W} \frac{d W}{d x}
\end{aligned}
$$




$$
\begin{gathered}
\frac{d Y_{i}}{d x}=\frac{\dot{\omega}_{i} W_{i} A}{\dot{m}}+\frac{1}{\dot{m}} \frac{d \dot{m}_{i, a d d}}{d x}-\frac{Y_{i}}{\dot{m}} \frac{d \dot{m}}{d x} \\
\frac{d W}{d x}=-W^{2} \sum_{i} \frac{1}{W_{i}} \frac{d Y_{i}}{d X}
\end{gathered}
$$

Equation (1) to equation (6) are the conservation of mass, the conservation of momentum, the conservation of energy, gas equation of state, the conservation of species and the conservation of molecular weight, respectively. The variables of A represents sectional area of the duct, $\mathrm{C}_{\mathrm{f}}$ represents skin-friction coefficient of the combustor duct, $\mathrm{cp}$ represents specific heat, $\mathrm{h}$ represents specific enthalpy, $\mathrm{Y}$ represents mass fraction, $\mathrm{W}$ represents molecular weight, $\varepsilon$ represents the ratio of injection velocity to mainstream velocity. The subscripts of $\mathrm{w}$ represents wall, aw represents adiabatic wall, add represents mass addition. The governing equations can be solved in the following form, referring to [8]:

$$
\begin{gathered}
\frac{1}{u} \frac{d u}{d x}=\frac{1}{\left(m a^{2}-1\right)}\left\{\begin{array}{c}
{\left[\frac{h_{t}}{C_{p} T}-k M a^{2}(1-\varepsilon)-1\right] \frac{1}{q_{m}} \frac{q_{m}}{d x}+\frac{1}{A} \frac{d A}{d x}+\frac{1}{W} \frac{d W}{d x}-k M a^{2} \frac{2 C_{f}}{D}-} \\
\frac{1}{C_{p} T} \frac{1}{q_{m}} \sum_{i}\left(h_{t . i} \frac{d q_{m}}{d x}\right)_{a d d}+\frac{q_{w} p_{w}}{T C_{p} q_{m}}+\frac{1}{C_{p} T} \sum_{i} h_{i} \frac{d Y_{i}}{d x}
\end{array}\right\} \\
\frac{1}{\rho} \frac{d \rho}{d x}=\frac{1}{q_{m}} \frac{d q_{m}}{d x}-\frac{1}{A} \frac{d A}{d x}-\frac{1}{u} \frac{d u}{d x} \\
\frac{1}{p} \frac{d p}{d x}=-k M a^{2}\left[\frac{1}{u} \frac{d u}{d x}+\frac{2 C_{f}}{D}+\frac{(1-\varepsilon)}{q_{m}} \frac{d q_{m}}{d x}\right] \\
\frac{1}{T} \frac{d T}{d x}=\frac{1}{p} \frac{d p}{d x}-\frac{1}{\rho} \frac{d \rho}{d x}+\frac{1}{W} \frac{d w}{d x} \\
\frac{d M a}{M a}=\frac{k}{2} \frac{d W}{W d x}+\frac{(k-1)}{2 C_{p}} \frac{d C_{p}}{d x}+\frac{1}{u} \frac{d u}{d x}-\frac{1}{2 T} \frac{d T}{d x}
\end{gathered}
$$

\subsection{Mixing}

After the fuel is injected into the combustor, the fuel has to be effectively mixed with air before it can burn. The calculation of mixing efficiency in supersonic flow refers to [2] and is listed as followed:

$$
\begin{gathered}
M_{c}=\frac{u_{f}-u_{a}}{a_{f}+a_{a}} \\
f\left(M_{c}\right)=0.25+0.75 e^{-3 M_{c}^{2}} \\
\left(\frac{L_{\text {mix }}}{d_{f}}\right)=\frac{K}{f\left(M_{c}\right)}\left[\frac{\rho_{f} U_{f}}{\rho_{a} U_{a}}\right]^{\frac{1}{2}} \\
\bar{x}=\frac{x-L_{\text {inj }}}{L_{\text {mix }}} \\
\eta_{\text {mix }}=1.06492\left(1-e^{-(3.69639 \bar{x})^{0.80586}}\right)
\end{gathered}
$$

Where the variables of $u_{f}$ and $u_{a}$ represents velocity of fuel injection and airflow, $a_{\mathrm{f}}$ and $\mathrm{a}_{\mathrm{a}}$ represents speed of sound in the fuel injector and airflow, $\mathrm{d}_{\mathrm{f}}$ represents the diameter of fuel injector, $\mathrm{L}_{\mathrm{inj}}$ represents the axis position of injector, and $\eta_{\text {mix }}$ represents the mixing efficiency. By solving the equations above, we can get the mixing efficiency that increases from none to maximum alongside $x$-axis.

Even though the method in [2] is raised for the calculation of the mixing between hydrogen and air, but we believe with few modifications of certain parameters, it can also be applied to the calculation of supercritical kerosene mixing.

\subsection{Modelling of supersonic shock wave mode}

When the equivalence ratio in the combustor reaches a certain level and the pressure in the combustor is high enough to cause boundary layer separation, the scramjet will work under supersonic shock wave mode. In this case, the separation of boundary layer in the combustor will trigger the forming of pre-combustion shock train in the isolator, which compresses the air and change the condition of airflow within the range of the precombustion shock train. The boundary layer separation in the combustor will change the shape of actual flow area and change the state of airflow in the combustor. Therefore, the modelling of supersonic shock wave mode is critical.

Through experimental research, the boundary of combustion mode transition is determined in [3]:

$$
\left(\frac{M_{d}}{M_{u}}\right)_{\text {turbulent }}<0.762
$$

Equation 17 shows that when the ratio of the minimum mach number in the combustor to the mach number at the entrance of the isolator is below 0.762 , the scramjet is considered to work under supersonic shock wave mode.

According to [4], the length of pre-combustion shock train can be calculated by:

$$
\begin{gathered}
L_{P C S T}=\frac{1}{c} \ln \left(\frac{w_{2}}{w_{3}}\right) \\
w^{2}=\frac{(k-1) M a^{2}}{2+(k-1) M a^{2}}
\end{gathered}
$$

Where the coefficient $\mathrm{c}$ can be determined by experiment, [5] suggest that $\mathrm{c}=0.114$.

Based on experimental research, [6] testified the theory that the ratio of the maximum pressure in the combustor to the pressure at the entrance of the combustor is a fixed number when the scramjet is working under supersonic shock wave mode. Which can be described as:

$$
p_{3}=\delta p_{\max , \mathrm{comb}}
$$

Where $\mathrm{p}_{\max , \mathrm{comb}}$ represents the maximum pressure in the combustor and $\delta$ is the ratio

Now that we have the values of pressure at the entrance of the isolator, the entrance of the combustor and the maximum pressure in the combustor, we can use polynomial fitting to get the whole pressure distribution, according to [7]. 


$$
\begin{gathered}
\frac{P(x)}{P_{2}}=1+\left(\frac{P_{3}}{P_{2}}-1\right)(3-2 \chi) \chi^{2} \\
\chi=\frac{x-x_{2}}{x_{3}-x_{2}} \\
\frac{P(x)}{P_{3}}=1+\left(\frac{P_{t}}{P_{3}}-1\right)(2-\chi) \chi \\
\chi=\frac{x-x_{3}}{x_{4}-x_{3}}
\end{gathered}
$$

Equation 21 and equation 23 are the polynomial fitting equations to solve the pressure distribution in the pre-combustion shock train and in the combustor under the influence of the separation of boundary layer, respectively.

\section{Cooling channel}

Cooling channels are placed around the combustor to absorb the heat from the combustor wall. Regenerative cooling can keep the combustor wall from over temperature while giving the fuel a certain amount of heat sink.

\subsection{Heat transfer}

The heat transfer between the cooling channel and the combustor wall includes both convective heat transfer and radiant heat transfer.

\subsubsection{Convective heat transfer}

According to [3], the heat flux density of convective heat transfer can be calculated in the following form:

$$
\begin{gathered}
\mathrm{r}=\sqrt[3]{\operatorname{Pr}^{*}}=\sqrt[3]{\frac{\mu^{*} C_{p}^{*}}{\lambda^{*}}} \\
h^{*}=\frac{h+h_{w}}{2}+0.22 r \frac{u^{2}}{2} \\
\operatorname{Re}_{x}=\frac{p u w x}{R T^{*} \mu^{*}} \\
q_{c}=\frac{0.0287 p u w\left(h_{a w^{-}}-h_{w}\right)}{\operatorname{RT}^{*} \operatorname{Pr}^{* \frac{2}{5}} \operatorname{Re}_{x}^{* \frac{1}{5}}}
\end{gathered}
$$

Where $\mu$ represents viscosity, $\mathrm{q}_{\mathrm{c}}$ represents the heat flux density of convective heat. Variables with superscript ' $*$ ' represent they are calculated under reference temperature. [3] suggest that $\operatorname{Pr}^{*}=0.9$. The skinfriction coefficient of the combustor duct can be calculated with the reference tempurature solved.

$$
C_{f}=\frac{0.0574}{\operatorname{Re}_{x}{ }^{*} \frac{1}{5}} \frac{T}{T^{*}}
$$

\subsubsection{Radiant heat transfer}

The radiant heat transfer between gas and combustor wall can be calculated as:

$$
q_{r}=\sigma \varepsilon_{w, e f}\left(\varepsilon_{g} T_{g, s}{ }^{4}-a_{w} T_{g, w}{ }^{4}\right)
$$

Where $\sigma$ represents Boltzmann's constant, $\varepsilon_{\mathrm{w}, \mathrm{ef}}$ represents effective blackness, $\varepsilon_{\mathrm{g}}$ represents theblackness of gas, $\mathrm{T}_{\mathrm{g}, \mathrm{s}}$ represents the temperature of gas, $\mathrm{T}_{\mathrm{g}, \mathrm{w}}$ represents the temperature of inner combustor wall.

The total heat flux density is:

$$
q_{w}=q_{r}+q_{c}
$$

\subsection{Flow in channel}

The basic idea of solving the flow identity in the cooling channel is the same as the solving in combustor. Here we directly put forward the solving equations, referring to [9].

$$
\begin{gathered}
\frac{d u_{f}}{d x}=\left(-\frac{A_{c} p_{f} q_{w} r_{c w}}{T_{f} C_{p f} q_{m f}}+p_{f} \frac{d A_{c}}{d x}-\frac{1}{2} \frac{f}{D_{h c}} q_{m f} u_{f}\right) /\left(q_{m f}-\frac{A_{c} p_{f}}{u_{f}}-\frac{A_{c} p_{f} u_{f}}{T_{f} C_{p f}}\right) \\
\frac{d T_{f}}{d x}=\left(\frac{q_{w} P_{w}}{q_{m f}}-u_{f} \frac{d u_{f}}{d x}\right) / C_{p f} \\
\frac{d p_{f}}{d x}=p_{f}\left(\frac{1}{T_{f}} \frac{d T_{f}}{d x}-\frac{1}{u_{f}} \frac{d u_{f}}{d x}-\frac{1}{A_{c}} \frac{d A_{c}}{d x}\right) \\
\frac{d \rho_{f}}{d x}=\rho_{f}\left(\frac{1}{\rho_{f}} \frac{d \rho_{f}}{d x}-\frac{1}{T_{f}} \frac{d T_{f}}{d x}\right)
\end{gathered}
$$

Where $r_{c w}$ represents the wetted perimeter of combustor wall, $\mathrm{q}_{\mathrm{mf}}$ represents the mass flow of the coolant fuel. Variables with the subsript ' $f$ ' represtet the sates of the coolant fuel. The temperature of the sombustor wall can be sloved useing basic heat conduct theory.

\subsection{Thermal cracking}

For the long chain hydrocarbon, when the temperature raises to a certain level, thermal cracking will happen and the long chain hydrocarbon will crack into hydrocarbon with shorter carbon chain. The temperature and pressure in the cooling channel is often beyond the cracking limit, and the thermal property of the coolant fuel changes severely due to thermal cracking. Therefore, the process of thermal cracking must be included in the modelling of hydrocarbon fuel scramjet. According to [9], the process of light thermal cracking of decane can be predicted by PDD model :

$$
\begin{aligned}
\mathrm{C}_{10} \mathrm{H}_{22} \rightarrow & 0.151 \mathrm{H}_{2}+0.143 \mathrm{CH}_{4}+0.256 \mathrm{C}_{2} \mathrm{H}_{4} \\
& +0.126 \mathrm{C}_{2} \mathrm{H}_{6}+0.23 \mathrm{C}_{3} \mathrm{H}_{6}+0.18 \mathrm{C}_{3} \mathrm{H}_{8} \\
& +0.196 \mathrm{C}_{4} \mathrm{H}_{8}+0.102 \mathrm{C}_{4} \mathrm{H}_{10}+0.171 \mathrm{C}_{5} \mathrm{H}_{10} \\
& +0.124 \mathrm{C}_{5} \mathrm{H}_{12}+0.195 \mathrm{C}_{6} \mathrm{H}_{12}+0.089 \mathrm{C}_{6} \mathrm{H}_{14} \\
& +0.169 \mathrm{C}_{7} \mathrm{H}_{14}+0.152 \mathrm{C}_{8} \mathrm{H}_{16}+0.012 \mathrm{C}_{8} \mathrm{H}_{18} \\
& +0.053 \mathrm{C}_{9} \mathrm{H}_{18}+0.003 \mathrm{C}_{9} \mathrm{H}_{20}
\end{aligned}
$$

The reaction rate constant can be calculated as:

$$
k_{A}=1.6 \times 10^{15} \times \exp \left(-\frac{263.7}{R T}\right)
$$


With the thermal cracking model and the calculation method of thermal cracking rate, we can generally predict the thermal cracking process of decane.

\section{Turbo pump}

Turbo pump is a critical part of expander cycle scramjet. It uses the extra heat from the combustor and charges the fuel. Turbo pump is an important part to keep scramjet work properly.

\subsection{Centrifugal pump}

The torque of centrifugal pump can be calculated as:

$$
M=\frac{d L}{d t}=\rho q\left(R_{2} c_{p 2}-R_{1} c_{p 1}\right)
$$

Where $\mathrm{M}$ represents torque, $\mathrm{L}$ represents moment of momentum, $\mathrm{R}$ represents the radium, c represents the velosity. Variables with the subsript ' $p 1$ ' represtet entrance of the pump, ' $\mathrm{p} 2$ ' represents the exit of the pump.

The lift of the pump can be calculated as:

$$
\begin{aligned}
H=H_{m} \frac{n^{2}}{n_{m}{ }^{2}} & =\left(k_{2} q_{M m}{ }^{2}+k_{1} q_{M m}{ }^{2}+k_{0}\right) \frac{n^{2}}{n_{M}{ }^{2}} \\
& =k_{2} q_{m}{ }^{2}+k_{1} q_{m} \frac{n}{n_{M}}+k_{0} \frac{n^{2}}{n_{M^{2}}}
\end{aligned}
$$

Where $\mathrm{q}_{\mathrm{m}}$ represents mass flow, $\mathrm{n}$ represents rotary speed, $\mathrm{n}_{\mathrm{M}}$ represents reference rotary speed. And $\mathrm{k}_{0}, \mathrm{k}_{1}$, $\mathrm{k}_{2}$ are paramters which can be solved by experiment or numerical calculation. For a given centrifugal pump, its lift can be directy calculated using equation 39 . Based on the design of turbo pump in [10], $\mathrm{k}_{0}=5.256, \mathrm{k}_{1}=1.16, \mathrm{k}_{2}=-$ $0.625, \mathrm{n}_{\mathrm{M}}=25000, \mathrm{q}_{\mathrm{m}}=1$.

\subsection{Turbine}

Consider the flow of the fuel in the turbine as an adiabatic expansion process:

$$
\left(\frac{P t_{1}}{P_{t 0}}\right)^{(k-1) / k}=T_{t 1} / T_{t 0}
$$

Where the variables with the subscript ' $t 0$ ' represents before the static blade, ' $t 1$ ' represents after the static blade and before the rotating blade, ' $\mathrm{t} 2$ ' represents after the rotating blade. The conservation of energy in the static blade of the turbine can be described by the conservation of stagnation enthalpy:

$$
\begin{gathered}
i_{o}{ }^{*}=i_{1}{ }^{*} \\
i_{0}{ }^{*}=c_{p} T_{0}{ }^{*}=c_{p} T_{0}+C_{0}{ }^{2} / 2 \\
i_{1}{ }^{*}=c_{p} T_{l}{ }^{*}=c_{p} T_{I}+C_{I}{ }^{2} / 2 \\
\frac{c_{1}{ }^{2}-c_{0}{ }^{2}}{2}=c_{p}\left(T_{0}-T_{1}\right)
\end{gathered}
$$

Where the variables with the superscript '*' represents stagnation. The isentropic expansion work of the turbine is:

$$
\begin{aligned}
& E_{a d}=i_{t 0}{ }^{*} i_{t 2}{ }^{*}=C p\left(T_{t 0}{ }^{*} T_{t 2 a d}{ }^{*}\right) \\
& \frac{T_{t 0}{ }^{*}}{T_{t 2 a d}{ }^{*}}=\left(\frac{P_{t 0}{ }^{*}}{P_{t 2}{ }^{*}}\right)^{\frac{k-1}{k}} \\
& E_{a d}=C p T_{t 0}{ }^{*}\left(1-\frac{1}{\left(\pi_{p}{ }^{*}\right)^{\frac{k-1}{k}}}\right)=E_{a d}{ }^{s}-c_{t 2}{ }^{2} / 2 \\
& P_{T}=\eta_{T} \dot{m}_{T} E_{a d}{ }^{s} \\
& M_{T}=P_{T} / \omega
\end{aligned}
$$

Where $E_{a d}$ represent the isentropic expansion work, $E_{a d}{ }^{s}$ represents the ideal isentropic expansion work, $M_{T}$ represents the torque of the turbine, $\omega$ represents the rotary speed.

\subsection{Turbo pump assembly}

As the turbine and the centrifugal pump are rigidity connected, and we ignore all time related terms, the transmission relationship between them is simple:

$$
\begin{gathered}
\omega_{T}=\omega_{P} \\
M_{P}=M_{T}
\end{gathered}
$$

\section{Result and conclusion}

\subsection{Result}

We test the model by setting the boundary conditions with the mach number of 2.7 , the temperature of $1160 \mathrm{~K}$, the pressure of $0.1 \mathrm{MPa}$. Assuming the pressure at the exit of the turbo to be $1 \mathrm{Mpa}$, the pressure in the fuel tank to be $0.5 \mathrm{Mpa}$, the fuel temperature to be $300 \mathrm{~K}$. The geometry structure of the scramjet is:

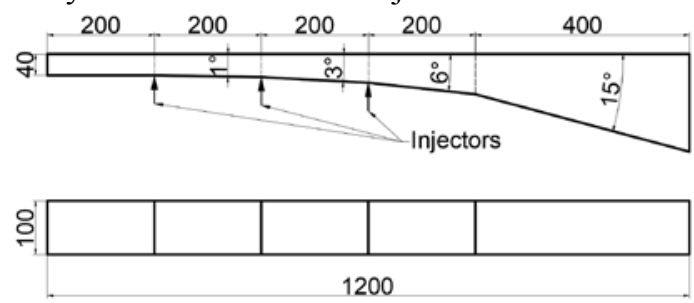

Figure 2. Geometry structure of the combustor.

The first $200 \mathrm{~mm}$ is the isolator, followed by the combustor, which consists of three 200mm-longexpanding sections. The expansion angle is 1 degree, 3 degree and 6 degree, respectively. Three fuel injectors are placed at the very front of the three expanding sections. At the end is the nozzle, which is $400 \mathrm{~mm}$ long and has a expansion angle of 15 degree.

\subsubsection{Example 1}


In the first example, all three fuel injectors inject fuel at the same mass flow with the total equivalence ratio of 0.6. At this state, the scramjet should be working under supersonic shock wave mode, according to equation 17 . We calculate the case using both pure supersonic combustion mode and supersonic shock wave mode, and the result is represented in blue and red line respectively, as is shown in Fig.3. By comparing the results, the influence of the pre-combustion shock train can be seen.
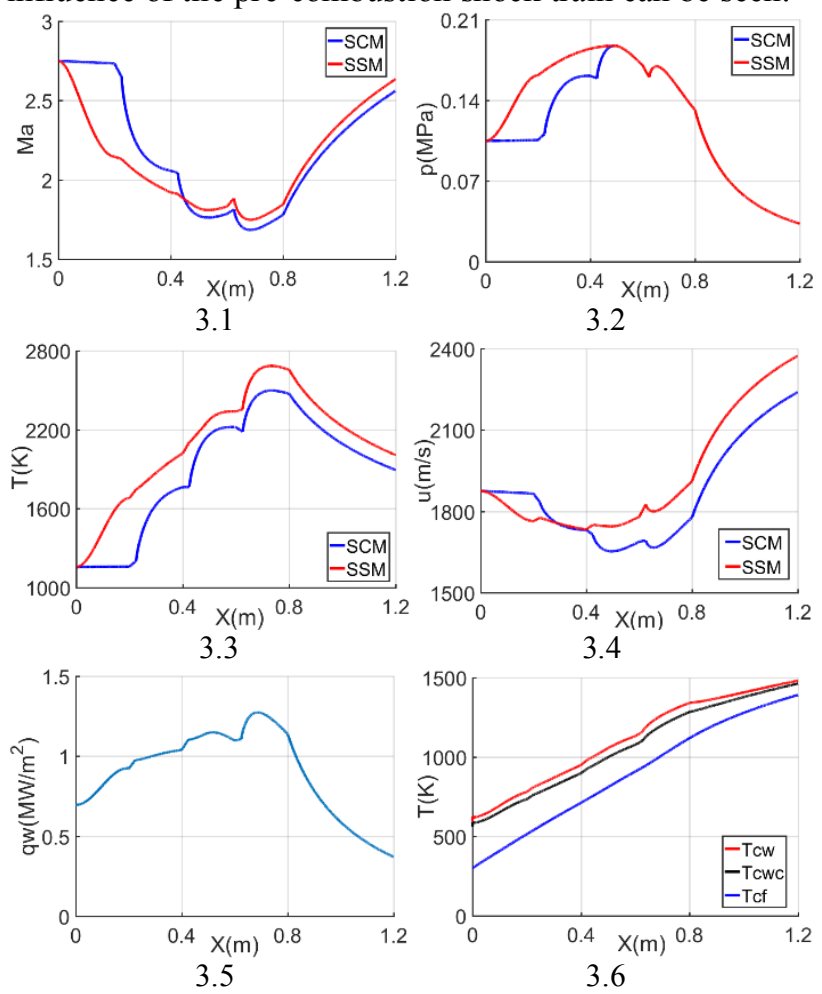

Figure3. Result of example 1.

Fig 3.1 to Fig 3.5 are the distributions of mach number, pressure, temperature, velocity, heat flux, respectively. Fig 3.6 shows the temperature distribution of combustor wall and coolant fuel, Tcw represents the inner side of combustor wall, Tcwc represents the outer side of combustor wall, Tcf represents the coolant fuel. Fig 3.5 and Fig 3.6 are calculated under shock wave mode.

According to the result of example 1, the model can correctly predict the influence of fuel injection, fuel combustion, heat transfer and the geometric change of the duct to the airflow. And the calculation of supersonic shock wave mode gives an expected result of the combustor flow field under the influence of precombustion shock train and boundary layer separation. The result shows that the temperature and velocity in supersonic shock wave mode is relatively higher than in pure supersonic combustion mode.

\subsubsection{Example 2}

The second example is to test how the rotating speed of turbo pump react to the increasing total equivalence ratio. Like example1, all fuel injectors inject fuel at the same mass flow. The result is shown in fig.4.

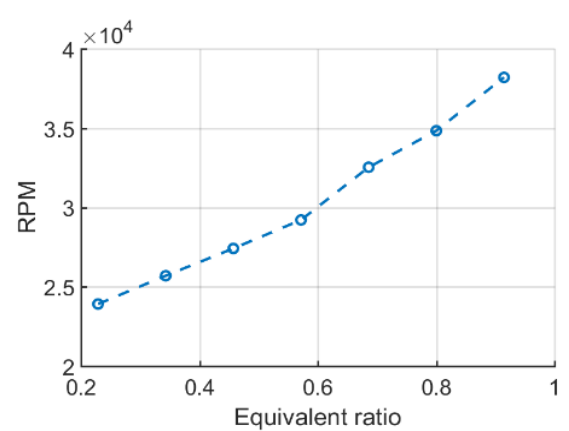

Figure4. Result of example 2 .

The rotary speed of turbo pump goes up with the increase of the total equivalence ratio, and the increasing rate of rotary speed increases after the scramjet entries supersonic shock wave mode.

\subsection{Conclusion}

A model of a kerosene based expander cycle scramjet is proposed. The simulation of the examples gives an expected result, and it have demonstrated that the model can predict the working condition of the expander cycle scramjet swiftly and correctly. With a few throttle devices added, it can be directly used in the simulation of the controlling process of the expander cycle scramjet. Moreover, by adding models of certain parts or processes to the model, it can be further modified. In the next step of work, the result of the model should be compared to experiment or numerical simulation and adjustment of parameters should be made to improve the accuracy of the model.

\section{References}

1. O'Brien, T. F., Starkey, R. P., and Lewis, M. J., "Quasi-One-Dimensional High-Speed Engine Model with Finite Rate Chemistry,"Journal of Propulsion and Power, Vol. 17, No. 6, 2001, pp. 1366-1374.

2. Birzer, C., and Doolan, C. J., "Quasi-OneDimensional Model of Hydrogen-Fueled Scramjet Combustors," Journal of Propulsion and Power, Vol. 25, No. 6, 2009, pp. 1220-1225.

3. Heiser, W., and Pratt, D., Hypersonic Airbreathing Propulsion, AIAA, Washington, D.C., 1994, pp. 479485.

4. Torrez S M, Dalle D J, Driscoll J F. New Method for Computing Performance of Choked Reacting Flows and Ram-to-Scram Transition[J]. Journal of Propulsion and Power, 2013, 29(2): 433-445.

5. Ikui T, Matsuo K, Nagai M. The Mechanism of Pseudo-Shock Waves[J].Bulletin of JSME, 1974, 17(108): 731-739.

6. Zhang Peng, Yu Gang, The study of one-dimensional flow analysis model of the combustor in supersonic combustion experiments [J]. Experiment and Measurements In Fluid Mechanics, 2003 (01): 88-92.

7. Billig $\mathrm{F}$ S, Dugger $\mathrm{G}$ L. Predictions of Precombustion Wall Pressure Distributions in Scramjet Engines [J]. Journal of Spacecraft and Rockets, 1973(9): 620-622. 
8. Zhang D, Feng Y, Zhang S, et al. Quasi-OneDimensional Model of Scramjet Combustor Coupled with Regenerative Cooling[J]. Journal of Propulsion \& Power, 2016, 32(3):1-11.

9. WARD T, ZABARNICK S, ERVIN J, et al. Simulations of flowing mildly-cracked normal alkanes incorporating proportional product distributions[J]. Journal of Propulsion \& Power, 2004, 20(3): 394-402.

10. Xuan Jin. Study on Operation Characteristics of Scramjet with Expansion Cycle. [D]. Changsha: National University of Defence Technology, 2016 